\title{
DEM examination of SPT correction factors
}

\author{
Ningning Zhang ${ }^{1,2 *}$, Marcos Arroyo $^{1}$, Matteo Ciantia ${ }^{3}$, and Antonio Gens ${ }^{1}$ \\ ${ }^{1}$ Division of Geotechnical Engineering and Geosciences, Department of Civil and Environmental Engineering, UPC Barcelona Tech, \\ 08034, Barcelona, Spain \\ ${ }^{2}$ Institute of Geotechnical Engineering, RWTH Aachen University, 52074 Aachen, Germany \\ ${ }^{3}$ School of Science and Engineering, University of Dundee, Dundee DD1 4HN, United Kingdom
}

\begin{abstract}
The Standard Penetration Test (SPT) is the most widely used method for dynamic testing of soils. The test is simple and robust but difficult to control and not fully standardized. As a result, experimental results typically show large variations and poor repeatability. To mitigate that correction factors such as energy normalization and rod length have been introduced in SPT practice. This study provides an examination of the two correction factors using models based on the discrete element method (DEM).
\end{abstract}

\section{Introduction}

The Standard Penetration Test (SPT) is the most widely employed example of dynamic probing. In SPT, a sampler positioned on the end of a boring rod is driven into the ground from the bottom of a borehole by striking the rod with a hammer. The number of hammer blows required to drive the sampler through a distance of $30 \mathrm{~cm}$ after an initial advance of $15 \mathrm{~cm}$ is recorded as $N$. SPT results are widely used for estimation of soil properties, evaluation of liquefaction potential, etc.

The measured blow count at a site is affected by instrument and procedural variability, such as drill equipment from different manufacturers, hammer configurations, rod length, etc. Many efforts have concentrated in improving test reliability and repeatability, i.e., its ability to reproduce blow counts using different drill systems under the same soil conditions, by applying correction factors to SPT data. Nowadays several correction factors including energy ratio $E R$, short rod correction factor $\lambda$ and overburden stress normalization factor $C_{N}$ have been widely accepted and included into some standards, i.e. UNEEN ISO 22476-3 (2005) [1].

The energy ratio is computed normalizing the energy delivered from the hammer to the rod -which is measured experimentally- by the theoretical driving energy of an SPT. Depending on various hammer types and other testing details, the energy ratio in practical field testing can vary in a wide range from $30 \%$ to $90 \%$. It has become common practice to normalize the blow count, considering the energy ratio delivered to obtain a standardized blow number $N_{60} \quad\left(=E R^{*} N / 60\right)$. The benefits of energy normalization to improve test repeatability have been documented empirically using field test results $[2,3]$.
The short rod correction factor was proposed based on field observations (e.g. [4]) of delivered hammer energy that apparently showed a reduction as rod length was diminished. This correction was later questioned, because it lacked a clear physical base, as in principle energy losses should increase with a longer transmission rod [5]. These ideas have been currently developed, mostly by empirical testing, into an alternative theory in which energy efficiency of the test decreases with the increase of rod length [6]. Physical experiments with SPT are cumbersome to set-up [6]. It is therefore interesting to further investigate the effect of rod length using numerical tools, e.g. discrete element method (DEM).

In this study, we attempt to validate the energy normalization and investigate the rod length effect using DEM-based models. In the following sections, we will first introduce the essential aspects of building DEM specimen and dynamic impact execution. We then calculate precisely the energy input for two series of dynamic tests examining the validity of different energy normalization and the effect of rod length, respectively.

\section{DEM model description}

Here in what follows we describe the essential details of the model set up presented in our previous work [9] for ease of reference. All the numerical models presented in this work are performed using the DEM code PFC3D [10].

\subsection{Fontainebleau sand analogue}

A discrete analogue of Fontainebleau sand was created using unbreakable spherical particles. Particle rotation was completely restricted to roughly mimic non-

\footnotetext{
* Corresponding author: n.zhang@geotechnik.rwth-aachen.de

A video is available at https://doi.org/10.48448/zpv5-jh18
} 
spherical particle shapes. This approach has been successfully used with angular granular materials in previous work [8], [11], [12].

Contacts between particles are set as elasto-plastic. Slip behavior at contacts is limited by a friction coefficient $\mu$. A simplified Hertz-Mindlin contact model is used to represent non-linear contact stiffness which is controlled by the elastic properties of the material grains, i.e. shear modulus, $G$, and Poisson's ratio $v$. The contact model properties $(G, \mu, v)$ (Table 1$)$ were taken from a calibration reported in the work of Ciantia et al., [13] for the same granular material.

Table 1. DEM contact model parameters.

\begin{tabular}{|c|c|c|c|}
\hline Material & $\boldsymbol{G}: \mathbf{G P a}$ & $\boldsymbol{\mu}$ & $\boldsymbol{v}$ \\
\hline F-sand & 9 & 0.28 & 0.2 \\
\hline Rod & 77 & 0.3 & 0.52 \\
\hline
\end{tabular}

\subsection{Chamber construction}

Fig. 1 shows the construction of a 3-dimensional virtual calibration chamber (VCC) to execute SPT. Table 2 gives the geometrical characteristics of the virtual calibration chamber. In order to obtain a manageable number of particles, the original sand particle sizes were scaled up by a factor of 79, thus achieving a reasonable rod/particle ratio $n_{p}=3.06$. The discrete analogue was filled into the chamber to specified relative density using the radius expansion method (REM). Isotropic compression to $5 \mathrm{kPa}$ in which inter-particle friction was reduced was used to attain a target porosity. After equilibration, inter-particle friction was reset to the calibrated value and isotropic stress was ramped up to a target level.

Table 2. Geometrical characteristics of the virtual calibration chamber.

\begin{tabular}{|c|c|c|}
\hline Variable (unit) & Symbol & DEM \\
\hline $\begin{array}{c}\text { Chamber diameter } \\
(\mathrm{mm})\end{array}$ & $D_{c}$ & 760 \\
\hline $\begin{array}{c}\text { Rod outside diameter } \\
(\mathrm{mm})\end{array}$ & $d_{c}$ & 50.8 \\
\hline $\begin{array}{c}\text { Chamber height (mm) } \\
\text { Rod length (m) }\end{array}$ & $H$ & 500 \\
\hline $\begin{array}{c}\text { mean element size } \\
\text { (mm) }\end{array}$ & $D_{50}$ & 16.6 \\
\hline $\begin{array}{c}\text { Chamber/rod diameter } \\
\text { ratio }\end{array}$ & $D_{c} / d_{c}=R_{d}$ & 15 \\
\hline $\begin{array}{c}\text { Rod/particle ratio } \\
\text { A }\end{array}$ & $d_{c} / \mathrm{D}_{50}=n_{p}$ & 3.06 \\
\hline
\end{tabular}

A flat-ended rod was created using a rigid closedended cylinder to mimic a plugged SPT sampling tube. The rod was firstly driven into the sample at a constant rate of $40 \mathrm{~cm} / \mathrm{s}$ until a depth of $15 \mathrm{~cm}$ was reached. A slight pull-back of the rod was performed before launching dynamic penetration, to avoid lock-in forces.

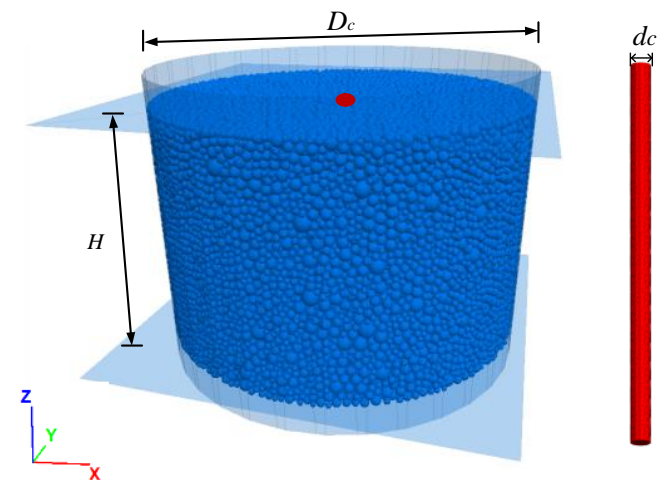

Fig. 1. View of DEM model of calibration chamber, rod and coordinate (originated at the center of the bottom wall)

\section{Dynamic driving}

\subsection{Methodology}

Dynamic driving was achieved by imposing on the rigid rod a pre-specified input force-time evolution. The timedependent input force $F_{d r v}$ (Fig. $2 \mathrm{a}, \eta_{d}=1$ ) was derived using a model proposed by Fairhurst [14] to approximately represent the input force characteristics of SPT hammer blow. The model assumes that at the $\mathrm{rod} /$ hammer interface, rebound waves from the upper hammer end transmit successive compression pulses of progressively reduced stress levels. The peak force occurs during the first pulse and is given by

$$
F_{\text {max }}=\frac{\eta_{d} a \sqrt{2 g h E \rho_{r}}}{1+\left(\frac{a}{A_{h}}\right)}
$$

Where, $\eta_{d}$ represents a dynamic efficiency ratio, the ratio of cross-sectional area of the rod, $a$, to the area of the hammer $A_{h}$, defines the hammer-rod impedance ratio $r$, if both the hammer and the rod are of the same material, $g$ is the gravitational acceleration, $h$ is the falling height of hammer, $E$ is the elastic modulus of the rod material and $\rho_{r}$ is the mass density of rod material.

The corresponding impact force $F_{n}$ for the $n$th $(n>1)$ compression pulse, is

$$
F_{n}=F_{\max }\left(\frac{1-r}{1+r}\right)^{n-1} \text { for } \frac{2(n-1) L}{c}<t<\frac{2 n L}{c}
$$

Where, $L$ is the hammer length, $t$ defines the time duration of each compression wave and $c$ is the wave propagation velocity.

The simulated impact is terminated at time $t_{\max }=2 \mathrm{l} / \mathrm{c}$ after the start of impact, where $l$ is the rod length.

Table 3. Parameters used for the simulated driving system.

\begin{tabular}{|c|c|c|c|c|}
\hline$\rho_{\boldsymbol{r}}: \mathbf{k g} / \mathbf{m}^{\mathbf{3}}$ & $\boldsymbol{E}: \mathbf{G P a}$ & $\boldsymbol{c}: \mathbf{m} / \mathbf{s}$ & $\boldsymbol{g}: \mathbf{m} / \mathbf{s}^{\mathbf{2}}$ & $\boldsymbol{l}: \mathbf{m}$ \\
\hline 8050 & 200 & 4984 & 9.8 & 10 \\
\hline $\boldsymbol{a}: \mathbf{m}^{\mathbf{2}}$ & $\boldsymbol{A}_{\boldsymbol{h}}: \mathbf{m}^{\mathbf{2}}$ & $\boldsymbol{r}$ & $\boldsymbol{L}: \mathbf{m}$ & $\boldsymbol{t}_{\max }: \mathbf{m s}$ \\
\hline 0.002 & 0.008 & 0.25 & 0.97 & 4 \\
\hline
\end{tabular}

The parameters describing the simulated driving system are collected in Table 3 . The default rod length 
is $10 \mathrm{~m}$. In this case, the SPT falling hammer (weight $m_{h}=63.5 \mathrm{~kg}$ and falling height $h=0.76 \mathrm{~m}$ ) will generate a 4 ms impact force with $F_{\max }=251 \mathrm{kN}$.

\subsection{Blowcount, blow energy and energy ratio}

The equivalent blow count $N$ is computed as the ratio of the reference $30 \mathrm{~cm}$ distance to the penetration depth per blow $\Delta \rho$. The transferred energy from the driven rod to the VCC in a given hammer blow, $E_{b l o w}$, is computed as the sum of hammer input work $W_{H}$ and work done by the rod self-weight, $U_{R}$. These energy terms can be calculated by integrating the work done by the impact force and gravitational forces on the driven rod,

$$
E_{\text {blow }}=W_{H}+U_{R}=\int_{0}^{t_{-} e q} F_{d r v}(t) v_{r}(t) d t+m_{r} g \int_{0}^{t_{-} e q} v_{r}(t) d t
$$

Where, $v_{r}(t)$ is the driven rod velocity history, which is an output of the test and the upper limit of the integral, $m_{r}$ is the rod mass, $t_{e q}$ is the time for equilibration.

The energy ratio $E R$ is computed with the ratio of the energy delivered and the theoretical maximum driving energy of an SPT (472 J)

$$
E R=\frac{E_{\text {blow }}}{m_{h} g h}
$$

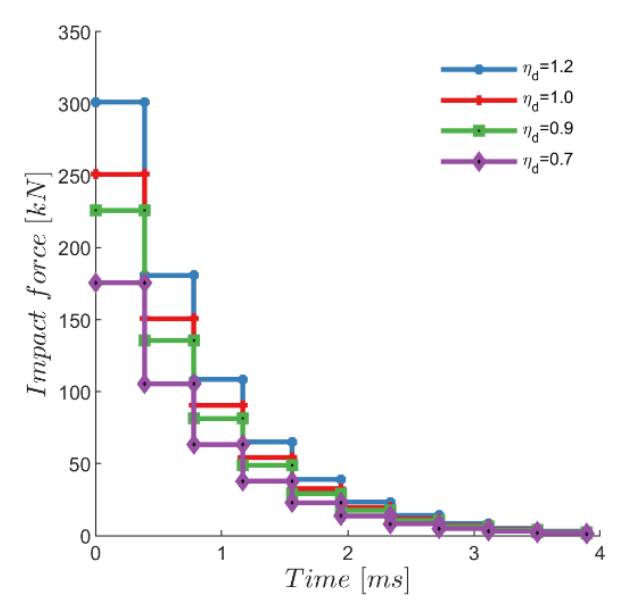

(a)

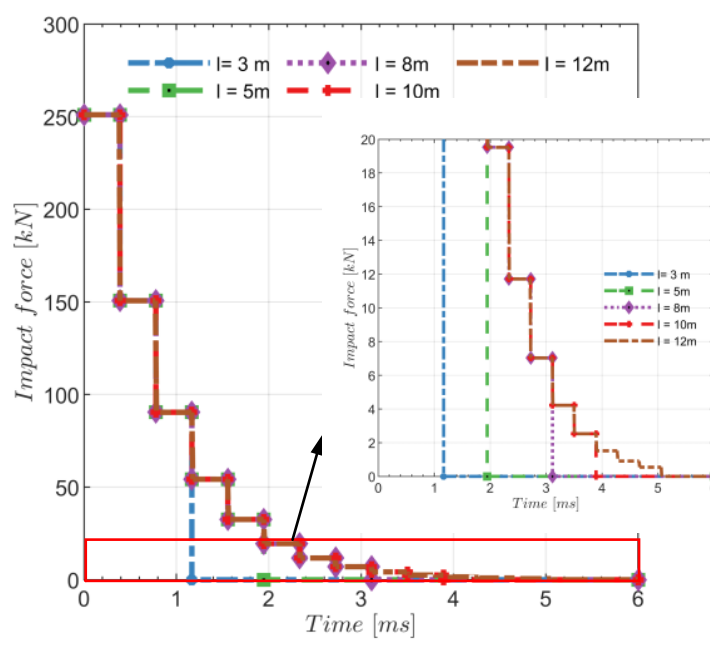

(b)

Fig. 2. Force-time configurations for (a) various dynamic efficiencies and (b) various rod lengths.

\subsection{Simulation program}

A dense specimen with the porosity of 0.382 confined by $100 \mathrm{kPa}$ was created. Based on the above described force-time signal, a series of impact tests were performed to examine the validity of the energy normalization commonly employed in SPT practice. The tests were run modifying the driving force history by using various values of dynamic efficiency coefficient $(0.7,0.9,1.0$ and 1.2). All the other settings remain constant and therefore the driving time was maintained at $4 \mathrm{~ms}$. The corresponding force-time configurations are shown in Fig. 2a.

The employed methodology of dynamic driving takes rod length into consideration in the term of impact time duration $\left(t_{\max }=2 l / c\right)$ as well as through the inclusion on the model of the rod as a microelement, whose inertia is dependent on the attributed rod length. Therefore it is possible to explore the correction of rod length effect commonly used in SPT practice. Based on the forcetime signal of the case $\eta_{d}=1$, another series of impact tests were run varying impact time $(1.2,2,3.2,4,4.8$ $\mathrm{ms})$ resulting from five rod length values $(3,5,8,10$ and $12 \mathrm{~m}$ ), respectively (note that rod buckling is excluded by design in the model and by standardized rod selection in practice). The maximum and subsequent driving forces remained unchanged before the specified termination of impact. The corresponding force-time configurations are shown in Fig. $2 b$.

\section{Results}

\subsection{Energy normalization of blowcount}

The blows at different energy $\left(\eta_{d}=0.7,0.9,1.0\right.$ and 1.2) were simulated. The relationship of both measured blowcounts, $N$, and normalized blowcounts $N_{60}$ against energy efficiency $E R$ are presented Fig. 3. It is evident that the energy normalization works well, with all the normalized $N_{60}$ values very close to one another. This verifies the implications that the energy normalized blowcount value is independent of the driving system characteristics or equivalently $N_{60}$ is only affected by soil properties (parameters and state), as verified empirically by field testing data.

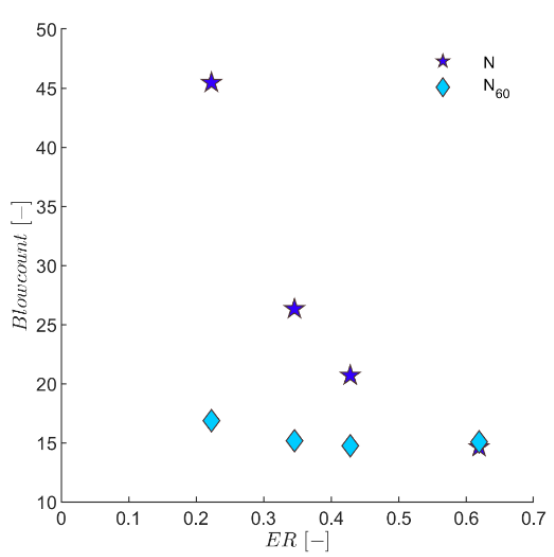

Fig. 3. Raw and normalized blowcounts versus energy ratio for various dynamic efficiencies. 


\subsection{Rod length effect on energy efficiency}

The blows on rods with various values of length $(l=3$, $5,8,10$ and $12 \mathrm{~m}$ ) were simulated. Fig 4a presents the effect of rod length on hammer impact energy $W_{H}$, rod potential energy $U_{R}$ and delivered energy $E_{\text {blow }}$ normalized by the theoretical maximum driving energy. With the increase of rod length, the hammer energy and the delivered energy to the soil both decrease, while the rod potential energy increases. These trends are in agreement with the twofold effect of rod length on energy efficiency identified by Odebrecht et al., [6]. On the one hand impact energy losses increase with increasing rod length, but, on the other hand, in a long rod composition the gain in potential energy from rod weight is significant and may partially compensate measured blow energy transmission losses.

Note that in the physical tests performed by Odebrecht et al [6] the rod was a composite one, whereas in the simulation is monolithic. The experimental losses in long rods were attributed to joint losses, whereas here those are not existing. What happens, instead, is that the inertial effects associated with a larger impactor overcome the benefits of a larger impact time. The rod length results in considerable discrepancy of blow count, but energy normalization is apparently able to alleviate the effect (Fig. 4b).

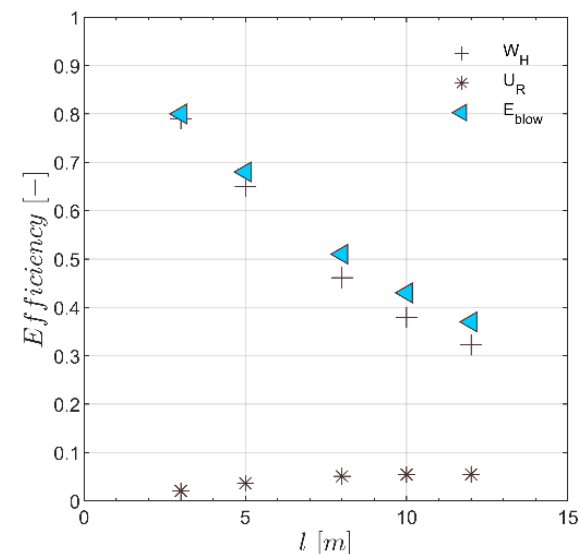

(a)

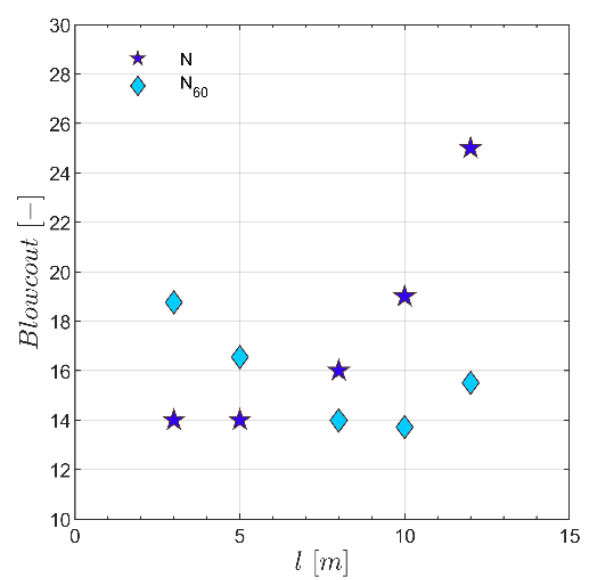

(b)

Fig. 4. (a) Hammer impact energy $W_{H}$, rod potential energy $U_{R}$ and delivered energy $E_{\text {blow }}$ normalized by the theoretical maximum driving energy versus rod length; (b) raw and normalized blowcounts versus rod length.

\section{Conclusions}

In this study, SPT was carried out numerically in a 3D virtual calibration chamber. The SPT energy normalization and rod length correction factors are examined from two series of impact set up varying energy efficiency coefficient and rod length. The main findings are

(1) Input energy normalization has been validated using DEM models to be as an effective approach as in field testing to improve test result repeatability.

(2) The twofold rod length effect observed by Odebrecht et al., [6] has been validated numerically. Energy transmitted decreases with increasing rod length. However, potential energy gained from rod weight is significant and does partially compensate for the dynamic losses.

\section{References}

[1] UNE-EN ISO 22476-3, Investigación y ensayos geotécnicos - Ensayos de campo - Parte 3: Ensayo de penetración estándar. (2005)

[2] A.W. Skempton, Géotechnique. 36, 3 (1986)

[3] H.B. Seed, K. Tokimatsu, L.F. Harder, R.M. Chung, J. Geotech. Eng. 111, 12 (1985)

[4] J.H. Schmertmann, A. Palacios, J. Geotech. Eng. Div. 105, 9 (1979)

[5] N. Aoki, J.C.A. Cintra, The application of energy conservation Hamilton's principle to the determination of energy efficiency in SPT test, in Proc., 6th Int. Conf. on the Application of Stress-Wave Theory to Piles, Rotterdam, The Netherlands (2000)

[6] E. Odebrecht, F. Schnaid, M.M. Rocha, G. de Paula Bernardes, J. Geotech. Geoenviron. Eng. 131, 10 (2005)

[7] T.T. Deger, Overburden Stress Normalization and Rod Length Corrections for the Standard Penetration Test (SPT), $\mathrm{PhD}$ thesis, University of California, Berkeley (2014)

[8] M. Arroyo, J. Butlanska, A. Gens, F. Calvetti, M. Jamiolkowski, Géotechnique. 61, 6 (2011)

[9] N. Zhang, M. Arroyo, M.O. Ciantia, A. Gens, J. Butlanska, Comput. Geotech. 111, 3 (2019)

[10] Itasca Consulting Group, PFC - Particle Flow Code, Ver. 5.0. Minneapolis (2017)

[11] M.O. Ciantia, M. Arroyo, J. Butlanska, A. Gens, Comput. Geotech. 73, 3 (2016)

[12] F. Calvetti, C. Prisco, R. Nova, J. Geotech. Geoenviron. Eng. 130, 12 (2015)

[13] M.O. Ciantia, M. Arroyo, C. O’Sullivan, A. Gens, T. Liu, Géotechnique. 69, 1 (2019)

[14] C. Fairhurst, Mine Quarr. Eng. 27, 3 (1961)

[15] F. Schnaid, E. Odebrecht, M.M. Rocha, G.D.P. Bernardes, J. Geotech. Geoenviron. Eng. 135, 8 (2009) 